\title{
Identical transacting factor requirement for knirps and knirps-related gene expression in the anterior but not in the posterior region of the Drosophila embryo
}

\author{
Mike Rothe†, Ernst A. Wimmer, Michael J. Pankratz, Marcos González-Gaitán, \\ Herbert Jäckle \\ Abteilung Molekulare Entwicklungsbiologie, Max-Planck-Institut für biophysikalische Chemie, Postfach 2841; Am Faßberg, D-37018 Göttingen, \\ Germany
}

(Received 22 December 1993; accepted 18 January 1994)

\begin{abstract}
The Drosophila genes knirps $(k n i)$ and knirps-related $(k n r l)$ are located within the 77E1,2 region on the left arm of the third chromosome. They encode nuclear hormone-like transcription factors containing almost identical $\mathrm{Cys}_{2} / \mathrm{Cys}_{2}$ DNA-binding zinc finger motifs which bind to the same target sequence. $k n i$ is a member of the gap class of segmentation genes, and its activity is required for the normal establishment of the abdomen. The function of $k n r l$ is still unknown; however, a possible gap gene function in the abdominal region of the embryo can be excluded. Both genes are initially expressed in three identical regions of the blastoderm embryo: in an anterior cap domain, in an anterior stripe and in a posterior broad band linked to the kni gap gene function. The transacting factor requirement for the expression of $k n i$ and $k n r l$ is identical for the two anterior domains but different, although similar, for the posterior domain of expression in the blastoderm. Both the anteroposterior morphogen bicoid and the dorsoventral morphogen dorsal are necessary but not sufficient for the activation of the two genes in the anterior cap domain, suggesting they act together to bring about its normal spatial limits.
\end{abstract}

Key words: Drosophila; Blastoderm; Segmentation genes; Spatial regulation

\section{Introduction}

The initial establishment of polarity along the two main axes of the Drosophila embryo is carried out by the maternal genes (St. Johnston and Nüsslein-Volhard, 1992, for review). A single genetic pathway is required for the development along the dorsal-ventral axis, while three distinct pathways (responsible for the development of the anterior, the posterior and the terminal regions of the embryo) are required for generating the body pattern along the anterior-posterior axis. The subsequent elaboration and refinement of spatial patterning along these two axes are executed by zygotic target genes (In- gham, 1988). The pattern elements along the anteriorposterior body axis are accomplished through a cascade of zygotic segmentation genes. Within this cascade, the gap genes are the first genes to be expressed in the embryo. The activity of this class of genes is required for the development of specific, overlapping sets of segment primordia. Terminal gap genes huckebein $(h k b)$ and tailless $(t l l)$ are needed to establish segmental primordia in the head and tail regions, and central gap genes such as hunchback ( $h b)$, Krüppel (Kr), knirps (kni) and giant $(g t)$ are required for segmentation in different positions of the trunk region of the embryo (Pankratz and Jäckle, 1993, for review). The segments that derive from a

†Present address: Molecular Biology Department, Genentech, Inc., 460 Point San Bruno Blvd., South San Francisco, CA 94080, USA. 
region covered by gap gene expression are affected by the corresponding mutations, e.g. gnathal and thoracic segments in the case of $h b$, and thorax and anterior abdominal segments in the case of $K r$.

The gap gene kni is expressed in two distinct regions of the early blastoderm embryo (Nauber et al., 1988). The posterior expression domain (posteriorly adjacent to the $\mathbf{K r}$ expression domain) is required for abdominal segmentation, while no mutant phenotype has yet been observed in the region corresponding to the anterior domain of kni gene expression (Nüsslein-Volhard and Wieschaus, 1980). Recently a second gene, knirpsrelated ( $k n r l)$, was identified (Oro et al., 1988, Rothe et al., 1989). $k n i$ and $k n r l$ are both located in the 77El,2 region on the left arm of the third chromosome (Rothe et al., 1989) and they are expressed in the same spatial and temporal patterns during early and late stages of embryogenesis. Furthermore, the putative $k n r l$ protein (KNRL) shows extensive sequence similarity in its DNA-binding region with the $k n i$ protein $(\mathrm{KNI})$ and both proteins can be grouped into the nuclear hormone receptor superfamily of transcription factors (Oro et al., 1988, Rothe et al., 1989). Moreover, KNRL can substitute $k n i$ segmentation function when expressed from a cDNA-derived minigene (Rothe et al., 1992), while in the wildtype embryo $\mathrm{knrl}$ is not involved in the establishment of abdominal segments. This lack of functional $k n r l$ activity is most likely due to the size of the $k n r l$ primary transcripts which contains three introns with a total length of $19 \mathrm{~kb}$ (Rothe et al., 1992). As the very rapid mitotic divisions in the early embryo allow only transcription of transcript of maximum 7-8 kb per nuclear division round, the kni transcript (which is only about $3 \mathrm{~kb}$ in length) can be full-sized transcribed, while the nascent $k n r l$ primary transcripts are aborted between the mitotic cycles (Rothe et al., 1992). This suggests that not enough or no knrl activity can accummulate during the developmental period shortly before, and during, blastoderm stage when the molecular prepattern of the segments is established in the abdominal region of the wildtype embryo (Rothe et al., 1992).

Here we show that the regulation of $k n i$ and $k n r l$ in their common anterior domains of expression requires the same transacting factors. Both genes fail to be activated in their anterior domains when either the anterior morphogen encoded by the maternal bicoid $(b c d)$ gene or the dorsal-ventral morphogen encoded by the maternal dorsal ( $d l)$ gene is lacking in the embryo. This suggests that the anterior expression domain requires the combined inputs from the maternal anteriorposterior and the dorsal-ventral pattern-forming pathways. In contrast to the identical control in the two anterior expression domains, expression of the two genes in their posterior expression domain is controlled by similar, though not identical, sets of transacting genes.

\section{Results and discussion}

The spatial expression patterns of the two genes $k n i$ and $k n r l$ are identical at the cellular blastoderm stage, but the way in which the expression patterns form is different (Fig. 1). With both genes, a posterior and two distinct anterior expression domains can be observed. We refer to the posterior circumferential band of kni or $k n r l$ gene expression as the 'posterior domain'. In the anterior, two distinct expression domains are formed by each gene. One of them forms a ventral cap extending from 70 to $100 \%$ egg length (EL), and we refer to it as the 'anterior cap domain'. The second forms a thin circumferential band at about $70 \%$ EL, overlapping with the anterior cap domain on the ventral side. We refer to it as the 'anterior stripe domain'. During gastrulation, both genes continue expression in their anterior domains. In contrast, expression in the posterior domain is transient and restricted to the blastoderm stage, i.e. both $k n i$ and $k n r l$ transcripts disappear from the posterior domain with the beginning of gastrulation.

To determine whether the temporal differences in the $k n i$ and $k n r l$ expression patterns might reflect different transacting control of gene activities for $k n i$ and $k n r l$, we have analysed $k n r l$ expression in Drosophila mutant embryos and compared it with the known spatial control for $k n i$ gene expression.

Fig. 1. Comparative analysis of blastodermal $k n i$ and $k n r l$ expression. Whole mount in situ hybridization of wildtype embryos with $k n i$ cDNA cJ15 (Nauber et al., 1988; left side) and $k n r l$ cDNA imd2 (Oro et al., 1988; right side). From top to bottom, subsequent stages of early development up to the beginning of gastrulation are shown. Orientation of embryos is anterior left, dorsal up. (a and b) Embryos prior to pole cell formation. Note the absence of localized $k n i$ and $k n r l$ expression. Note that during syncytial blastoderm ( $c$ and d) $k n i$ is first expressed in the posterior domain (c) while $k n r l$ expression is found in the anterior cap domain (d). Note that the embryo shown in (d) is older than the one shown in (c). During cellularization ( $e$ and $n k n i$ expression is also observed in the anterior cap (e), and $k n r l$ transcripts appear in the posterior domain (f; arrowhead). During a later stage of cellular blastoderm formation, the anterior stripe of expression occurs both with $k n i$ and $k n r l(g$ and $h$; anterior arrowhead indicates the position of the anterior stripe at the dorsal side of the embryo). Note that the posterior domain of $k n r l$ expression (h; posterior arrowhead) is still weak when compared with the corresponding expression domain of kni (g) when the anterior cap domain is used as an internal marker. At a late stage of cellular blastoderm ( $\mathrm{i}$ and $\mathrm{k}$ ) both $\mathrm{kni}$ and $\mathrm{knrl}$ show high level of expression in each of the three expression domains. At an early stage of gastrulation ( 1 and $\mathrm{m}$ ) $k n i$ and $k n r l$ transcripts remain at high levels in the two anterior domains but they decrease in the posterior domain ( $m$; arrowhead). 


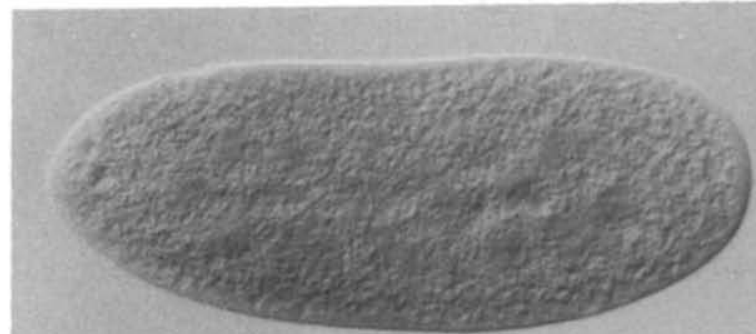

a
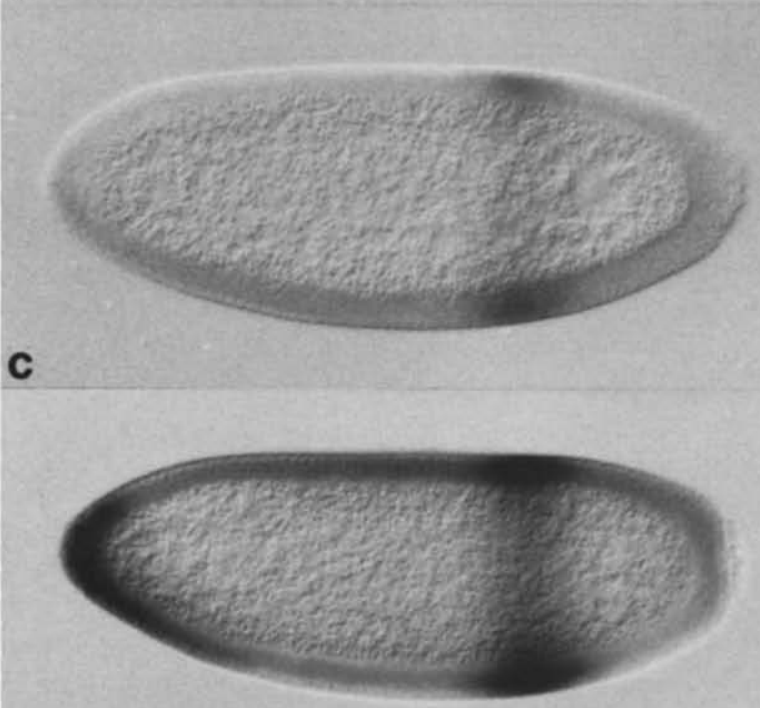

e

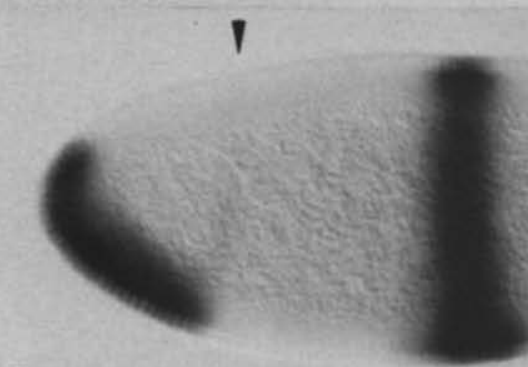

g

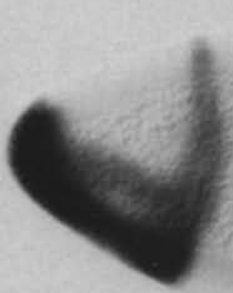

i

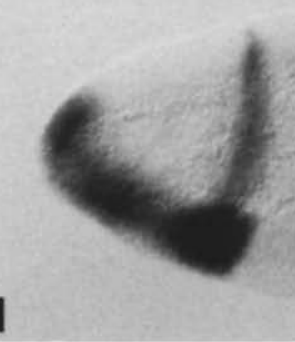

b

d

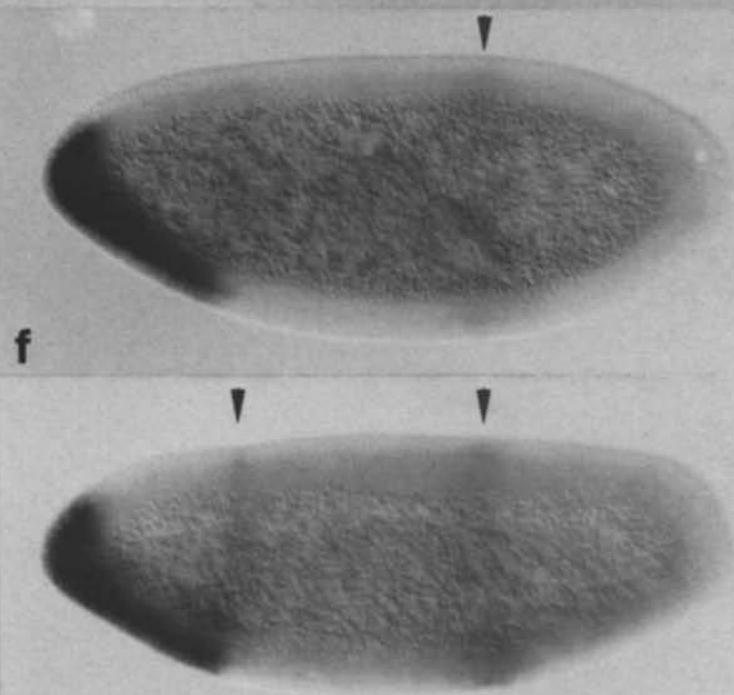

h

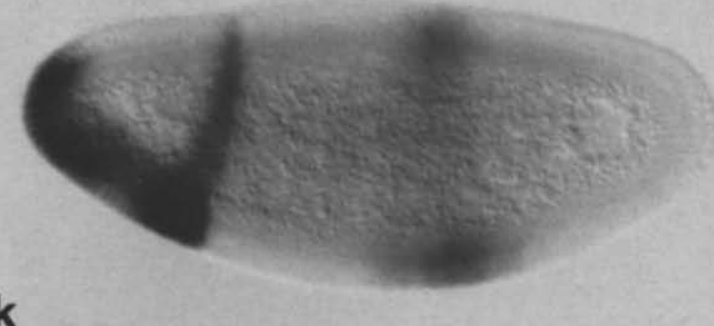

I

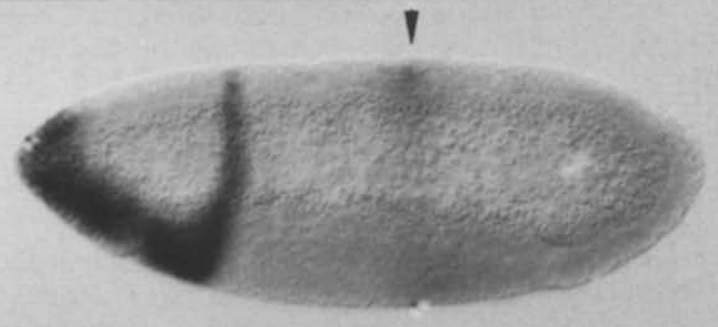

m 


\subsection{Spatial control of the knrl and kni posterior domains}

Recent analysis has shown that a modular array of regulatory units confers spatially distinct patterns of $k n i$ gene expression in the two distinct regions of the early embryo. With the cis-acting sequences required for the posterior domain of expression, kni can be activated throughout the wildtype embryo. The posterior domain of expression is formed through repression by $h b$ activity from the anterior and $t l l$ activity from the posterior (Pankratz et al., 1992). In addition, it has been reported that the gap gene $g t$ functions as a repressor in order to delimit the posterior border of kni gene expression (Eldon and Pirrotta, 1991; Kraut and Levine, 1991). On top of ubiquitous $k n i$ activation, $K r$ formally acts as an additional $k n i$ activator either directly or indirectly through repression of $g t$ (Pankratz et al., 1989; Capovilla et al., 1992).

The spatial limits of the posterior domain of $k n r l$ expression coincide with those of the kni posterior expression domain, but two differences can be noted. $k n r l$ appears first in the anterior cap and then in the posterior domain, whereas $k n i$ is first expressed in the posterior domain and shortly thereafter in the anterior cap (compare Fig. lc and d, le and f). Furthermore, the level of $\mathrm{knrl}$ expression in the posterior domain is always lower than in the anterior, except during late cellular blastoderm stage, while the level of expression of kni is about the same in both domains. These minor differences suggest that the transacting requirement for $k n r l$ expression in the posterior domain may differ from that of $k n i$.

Maternal control. We have previously shown that the posterior expression domain of $k n i$ and $k n r l$ is absent in embryos which lack the nanos gene product (Fig. 2a, b, f and g; Rothe et al., 1989). As nanos activity prevents maternal $h b$ expression in the posterior region of the embryo it is likely that the presence of $h b$ activity in the posterior region of nanos mutant embryos causes repression of both $k n i$ and $k n r l$ gene expression, and that $h b$ acts as a direct repressor of both genes (Pankratz et al., 1992; see also below). In the absence of the anterior maternal organizer system, such as in embryos laid by $b c d$ mutant females, the posterior expression domain of both genes is shifted towards the centre of the embryo but otherwise is not affected (Fig. 2c and h). Thus, the expression of both genes in bcd embryos follows the known shift of the anlagen plan towards anterior. This suggests that the anterior maternal organizer system has either no direct influence on the posterior domain of expression of the two genes, or that it is part of a redundant activator system. In the absence of the terminal maternal organizer system, such as in embryos laid by torso $^{\mathrm{PM}}\left(\right.$ tor $\left.^{\mathrm{PM}}\right)$ females, the posterior domain of kni expression expands into the posterior pole region while $k n r l$ expression is left unaffected (Fig. 2d and i). This suggests that the posterior terminal system represses $k n i$ expression in wildtype embryos while the posterior border of $k n r l$ expression in the posterior domain is set by a different mechanism than with $k n i$ (see also below). Taken together, these results show that the posterior expression domain of both $k n i$ and $k n r l$ cannot be activated solely under the control of the anterior organizer system, and that the activation of both genes requires the absence of repressing $h b$ activity in the posterior region of the embryo through the activity of the posterior maternal organizer system. However, a major difference between the two posterior expression domains is that tor-dependent genes are required to set the posterior limit of the kni expression domain while $k n r l$ appears to be independent of tor-dependent activities.

Gap gene control. Activation of kni expression in the posterior domain depends on a global activator system, and the activity of $K r$ is required to enhance or coactivate kni expression (Pankratz et al., 1989; 1992). This mode of regulation results in a very transient $k n i$ expression domain in $\mathrm{Kr}$ mutant embryos. kni disappears at the late cellular blastoderm stage in $K r$ mutant embryos while it disappears only during early gastrulation in wildtype embryos (Fig. 3a and b). In contrast to $k n i$, the posterior $k n r l$ expression domain cannot be observed in $K r$ mutant embryos (Fig. 3e and f). This suggests that $K r$ directly or indirectly activates $k n r l$ expression whereas $k n i$ is only partly dependent on activating $\mathrm{Kr}$ activity, i.e. $\mathrm{knrl}$ expression is not activated by a global activator system as is $k n i$, but rather by a single activator which is provided through $K r$ activity.

The anterior border of the kni and knrl posterior expression domains depends on $h b$ activity. In $h b$ mutant embryos, the posterior expression domain of both genes expands towards anterior (Fig. $3 \mathrm{c}$ and g). Thus, $h b$ activity is required to prevent $k n i$ gene expression in the anterior region of the embryo. This result is consistent with the finding that ubiquitous maternal $h b$ activity, as found in nanos mutant embryos, prevents both $k n i$ and $\mathrm{knrl}$ gene expression. A difference, with respect to the requirement of zygotic gene activities between the two genes, might be that the gap gene $g t$ represses kni (Eldon and Pirrotta, 1991; Kraut and Levine, 1991) while knrl expression is not affected in $g t$ mutants (data not shown). We note, however, that we have not observed a significant expansion of $k n i$ expression in $g t$ mutant embryos, leaving undetermined if $k n i$ is actually repressed by $g t$ in the wildtype situation. However, the spatial limit of the kni expression domain towards posterior clearly depends on $t l l$ activity, while an expansion of the posterior $k n r l$ expression domain has not been observed in $t l l$ mutant embryos (Fig. 3d and h). This indicates that the posterior domain of $k n i$ expression is delimited by $t l l$ activity which represses kni expression in the posterior region of the embryo, while the spatial limits of the posterior domain of $\mathrm{knrl}$ expression is not dependent on tll activity. In embryos where ectopic $t l l$ activity has 
kni $k n r l$

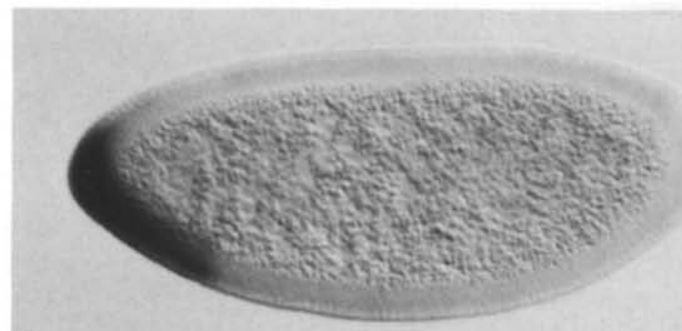

a

f

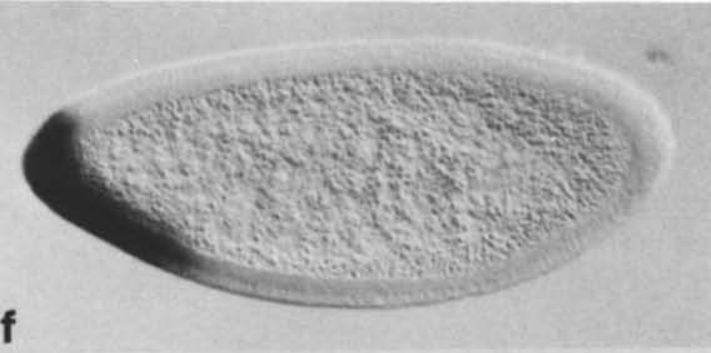

nos $\mathrm{L} 7$

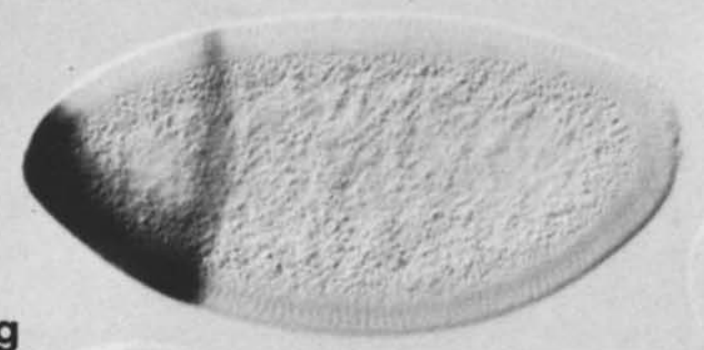

b

g
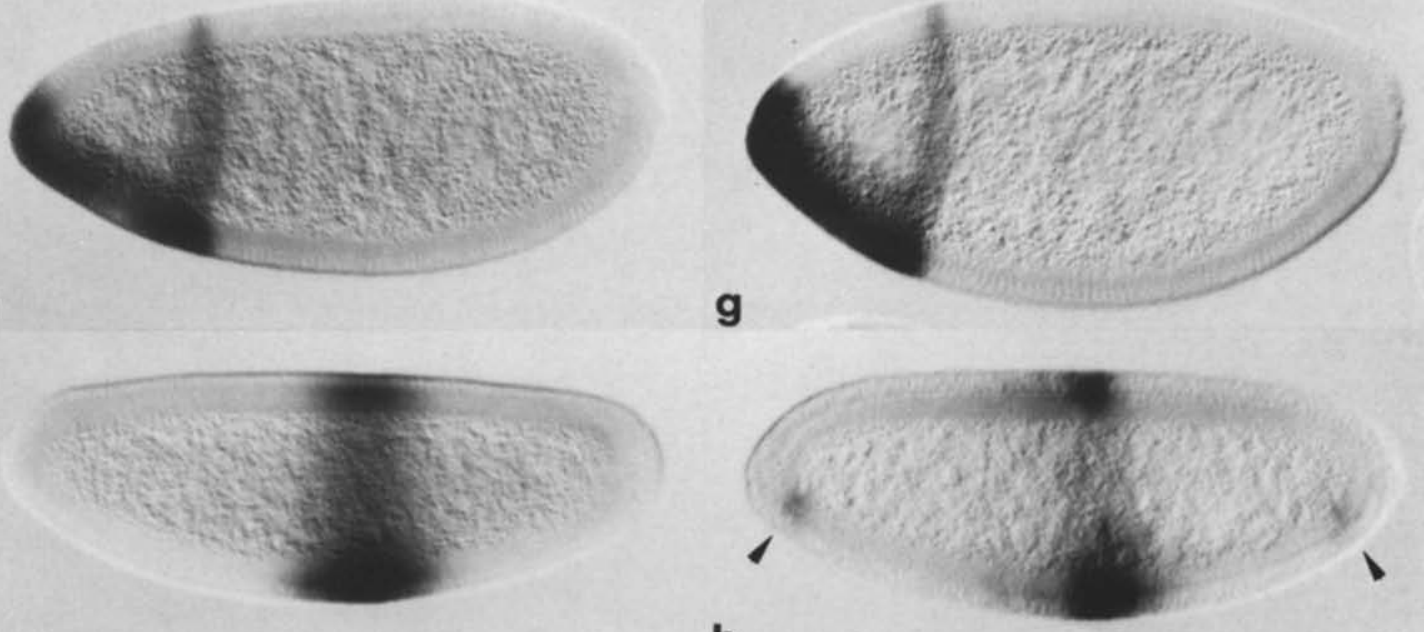

C

h

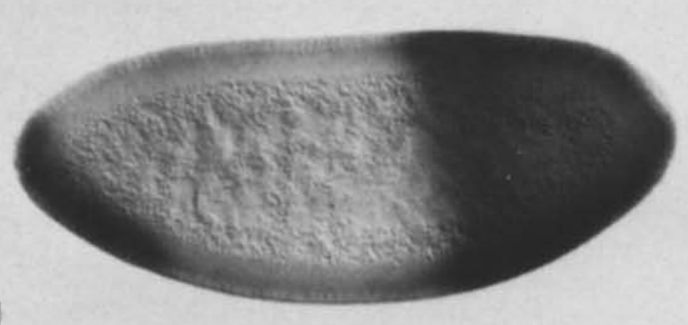

d

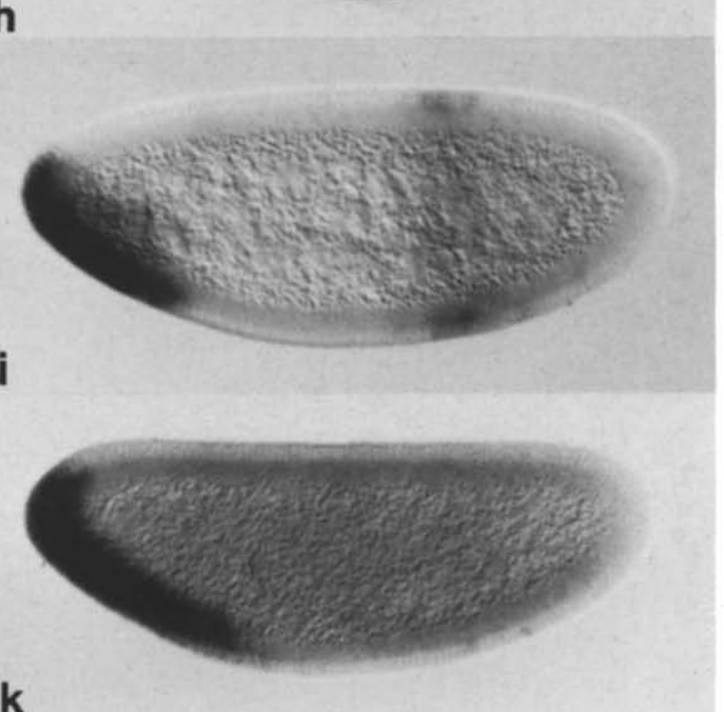

i

e

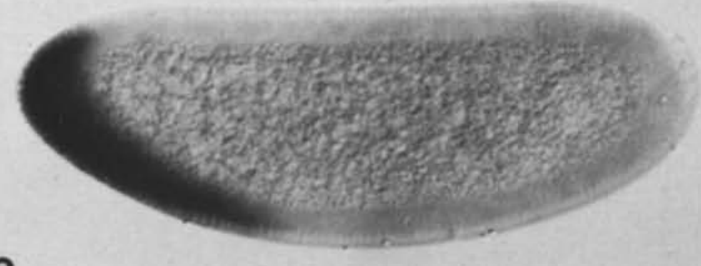

k

Fig. 2. $k n i$ and $k n r l$ expression is under the control of maternal gene products. (a-e) Embryos hybridized with a kni probe, (f-k) embryos hybridized with a $\mathrm{knrl}$ probe as described in Fig. 1 . The genotype of mutant females from which embryos derived is indicated on the left side. Embryos lacking the nos gene product are shown in ( $\mathrm{a}, \mathrm{b}, \mathrm{f}$ and $\mathrm{g}$ ). Note the absence of both $k n i$ and $k n r l$ posterior expression at an early (a and $\mathrm{f}$ ) and a late (b and g) blastoderm stage. ( $($ and $h$ ) Embryos lacking bcd activity. Note the absence of the anterior domains of kni and knrl expression and the appearance of a ventral dot of $k n r l$ expression in both the anterior and posterior region (h; arrowheads). (d and i) Expansion of the posterior domain of $k n i$ expression in embryos lacking tor activity. Note the posterior expansion of $k n i$ expression and the normal posterior expression domain of $k n r l$. (e and $\mathrm{k}$ ) Embryos laid by females carrying a dominant tor mutant gene which leads to ectopic activation of the tor-dependent terminal signalling pathway throughout the embryo. Note the absence of both the posterior domain of kni and knrl expression and an expansion of the anterior cap domain in both cases. For details, see text. Orientation of embryos is as described in Fig. 1. 

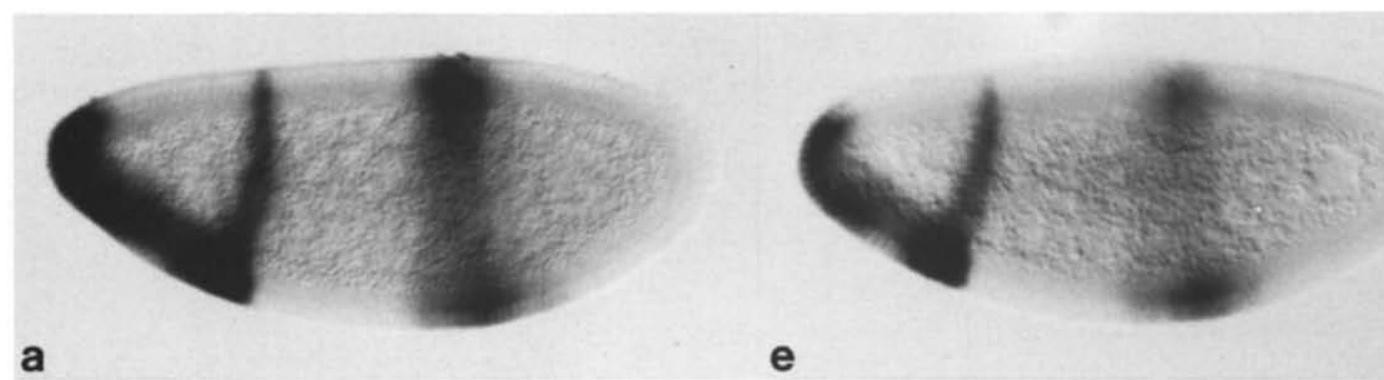

a

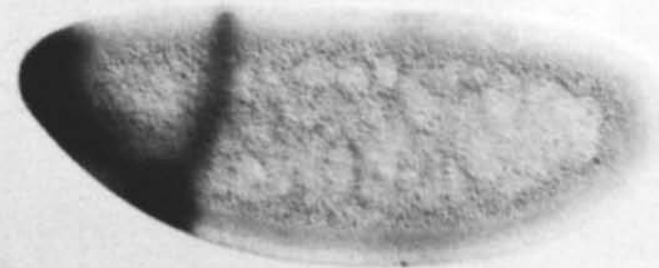

b

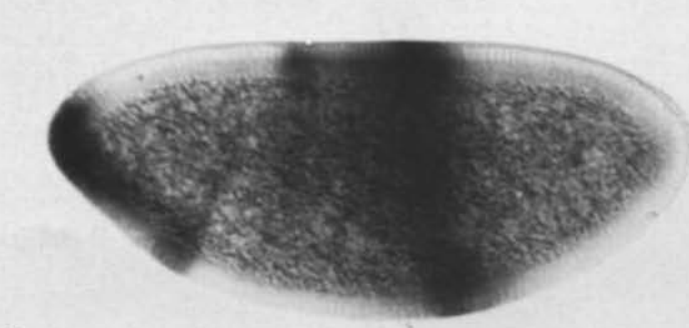

C

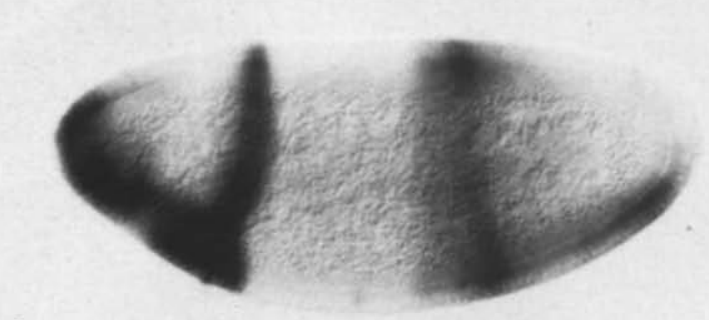

d

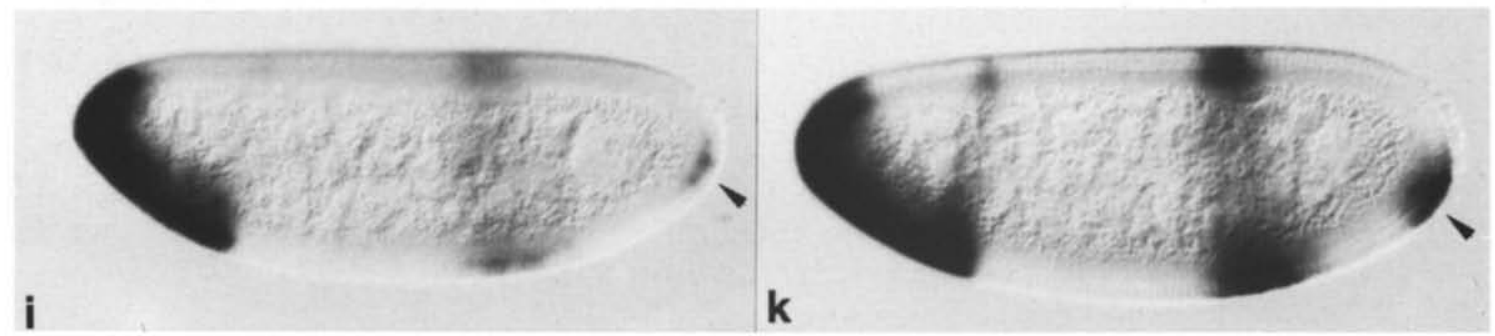

e

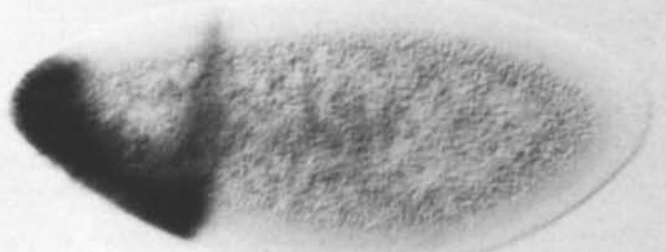

f

g
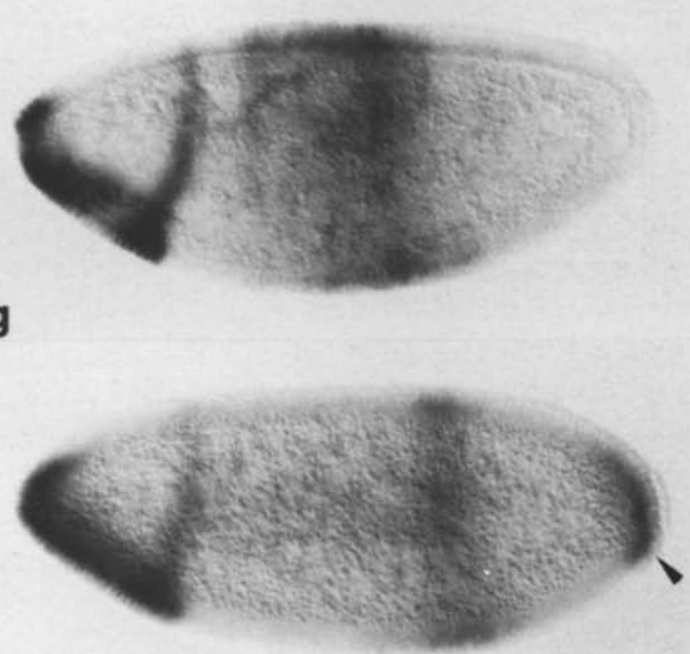

h

Fig. 3. $k n i$ and $k n r l$ expression in wildtype and gap mutant embryos at late blastoderm stage. (a-d) $k n i$ expression and (e-k) $k n r l$ expression monitored by whole mount in situ hybridization as described in the legend of Fig. 1. (a and e) Wildtype embryos; (b and f) homozygous $K r^{1}$ mutant embryos; (c and g) homozygous $h b^{9 \mathrm{Q}}$ mutant embryos; (d and h) homozygous $t l^{\mathrm{B}}$ mutant embryos. Note that $k n i$ but not $k n r l$ expression in the posterior region of the embryo is affected by the absence of the $t l l$ gene product. In order to identify the homozygous $t / l{ }^{8}$ mutant embryos in (h), double staining was performed involving the fork head $(f k h)$ cDNA probe (Weigel et al., 1989). Note the reduced $f k h$ expression domain in the posterior pole region of the embryo (arrowhead) indicating the $t l l$ homozygous state of the embryo. (i and $\mathrm{k}$ ) Double mutant homozygous $k n i^{301}{ } l^{8}$ mutant embryos hybridized with the $k n r l$ probe. Note that the posterior ventral spot of expression, which is not observed in wildtype embryos, cannot be observed with the kni probe. Note that the posterior domain of $k n r l$ expression is enhanced (due to the absence of $k n i$ activity) but not expanded in the double mutant embryo. This observation supports the finding that $t l l$ is not required to suppress $k n r l$ expression in the wildtype situation. 
been observed throughout the entire embryo, as in those laid by females containing the dominant tor $^{\text {D4021 }}$ allele, both $k n i$ and $k n r l$ are not expressed in the posterior domain (Fig. 2e and k). $t l l$ is likely to directly repress $k n i$ gene expression in the posterior region of the embryo (Pankratz et al., 1992). However, in the case of $k n r l$ the lack of expression could be caused by the absence of the activating $K r$ function. This proposal is consistent with the observed repression of $K r$ expression which occurs in the presence of ectopic $t l l$ activity in tor $^{\mathrm{D} 4021}$ embryos (Steingrimsson et al., 1991).

$k n i$ expression occurs earlier than $k n r l$ expression in the posterior region of the embryo. Therefore it is possible that $k n i$ activity might, with $K r$, activate $k n r l$ expression. In the absence of $k n i$ activity, $k n r l$ expression is normal at its initial stage (Fig. 4a and b). During cellular blastoderm stage, however, $k n r l$ expression increases significantly when compared to wildtype embryos (Fig. $4 c$ and d). Thus, $k n i$ activity does not activate but rather represses posterior $\mathrm{knrl}$ expression in wildtype embryos. To determine whether $k n i$ activity has an autoregulatory input on its own expression, we used a transgene in which the cis-regulatory region of the $k n i$ gene was fused to the lac $Z$ reporter gene. This construct conducts $\beta$ galactosidase expression in the authentic kni anterior cap and posterior expression domain (Fig. 5). In wildtype embryos, lac $Z$ reporter gene expression in these domains occurs with similar intensities. In kni mutant embryos, however, $\beta$-galactosidase expression in the posterior domain continues in the mutant embryos when it is already absent from wildtype, and the expression is significantly increased (Fig. 5). These results suggest that $k n i$ activity not only represses posterior $k n r l$ expression but also regulates its own activity. This negative autoregulatory effect of $k n i$ has already been suggested by Howard (1990).

Differential RNA-polymerase II requirement. kni expression is not only dependent on the activity of the segmentation gene cascade but also on more general factors, such as that characterized by wimp, a neomorphic or antimorphic mutation of the $140 \mathrm{kDa}$ subunit of RNA-polymerase II (Parkhurst and Ish-Horowicz, 1991). wimp interacts specifically with a series of maternal and zygotic genes by reducing their transcriptional activities (Parkhurst and Ish-Horowicz, 1991). In the case of $k n i$, the wimp mutation causes a reduced level of $k n i$ expression (Fig. 6a and b); embryos heterozygous for $k n i$ which were laid by wimp females develop a segmentation defect similar to the phenotype of homozygous kni mutant embryos (Parkhurst and IshHorowicz, 1991). By contrast, $k n r l$ expression appears enhanced in the posterior domain of embryos laid by wimp females (Fig. 6c and d). This enhancement is likely to be due to the decreased level of $k n i$ expression which results in less efficient repression of $k n r l$ by $k n i$ activity. Thus, wimp is unlikely to interact with $k n r l$. This sug- gests that the two genes are differently affected by the mutant $140 \mathrm{kDa}$ subunit of the RNA-polymerase.

Taken together, these results show that expression of $k n i$ and $k n r l$ in the posterior region of the embryo is controlled by a similar, though not identical, set of transacting regulators (summarized in Fig. $7 \mathrm{a}$ and b). The

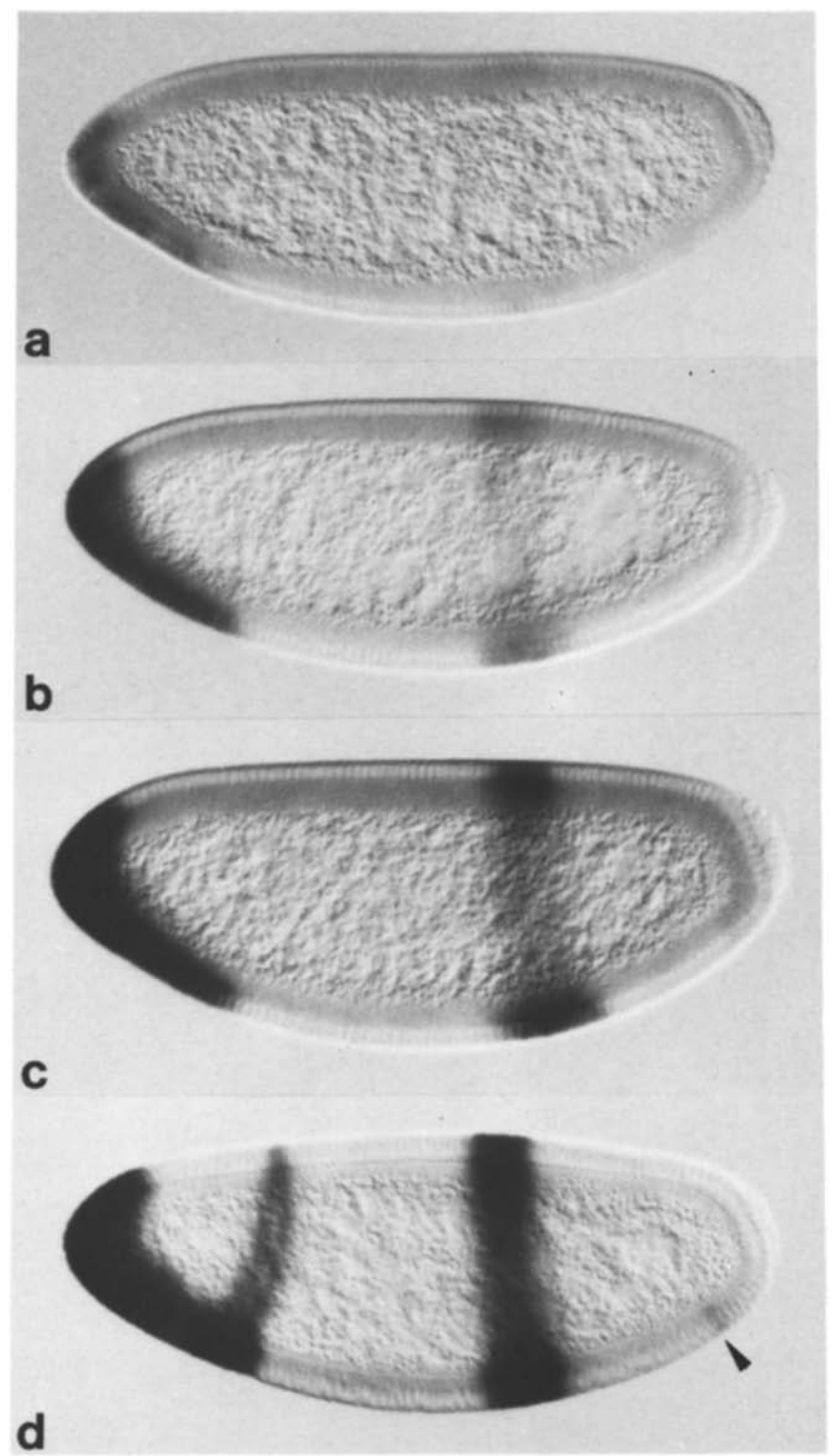

Fig. 4. Expression pattern of $k n r l$ in the blastoderm of $k n i$ mutant embryos. Embryos that derived from $k n i{ }^{\mathrm{FCl}}{ }^{3} / \mathrm{TM} 3 h b$-lacZ parents were hybridized with a combined probe consisting of $k n r l c D N A$ and $l a c Z$. As a result, homozygous $k n i^{\mathrm{FCl} 3}$ embryos could be distinguished from heterozygous $k n i^{\mathrm{FCl} 3}$ and those which are homozygous for the TM3, hb-lacZ embryos by the absence of lac Z expression. (a-d) Early to late blastoderm stages of $k n i^{\mathrm{FCl}}$ mutant embryos showing that $k n r l$ expression in the posterior region of the embryo is very strong (d) at a stage where knrl expression decays in wildtype embryos (see Fig. 1). Note the appearance of the ventral spot in the posterior region of the embryo shown in (d; arrowhead). 


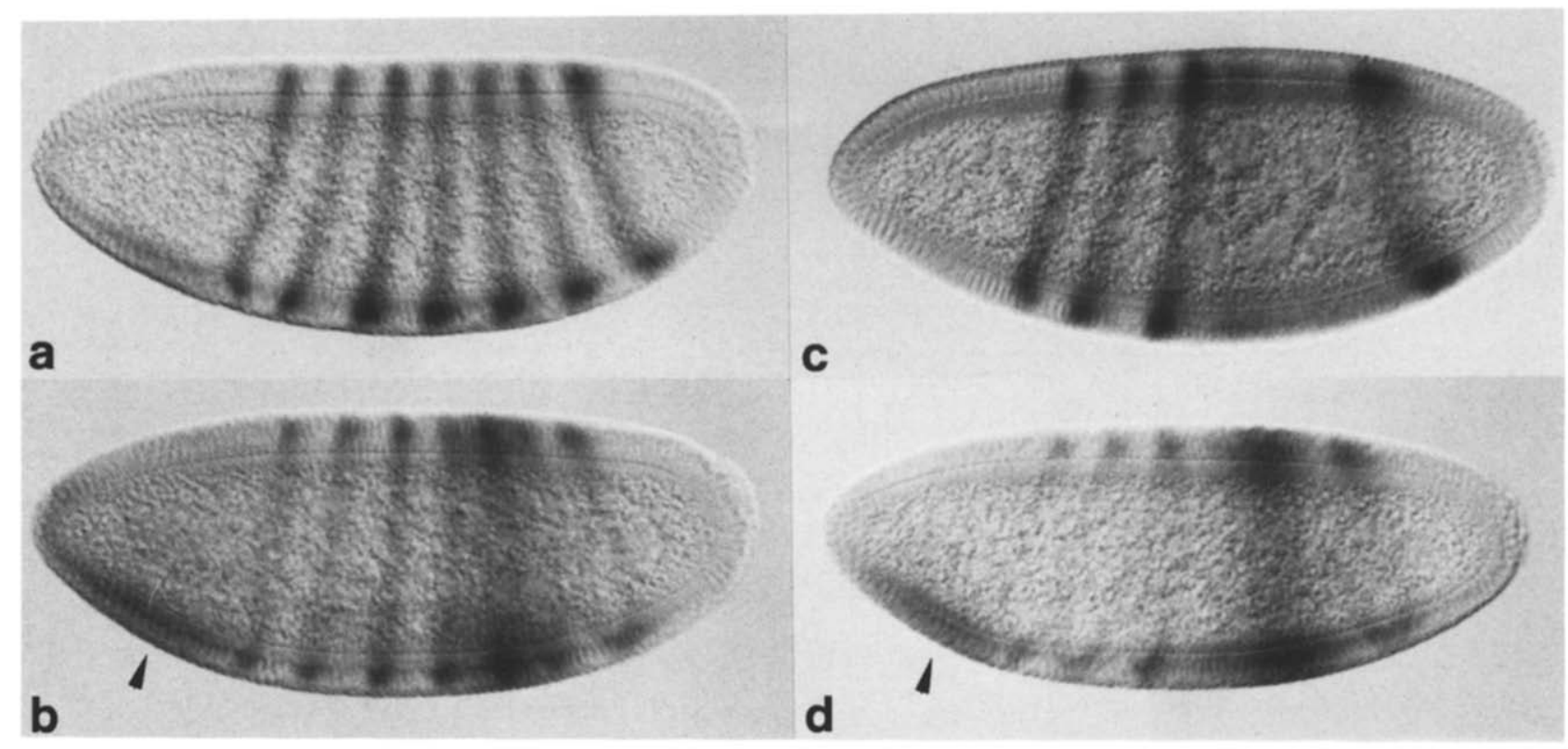

Fig. 5. Expression pattern of $\beta$-galactosidase under the control of the $k n i$ cis-acting sequences in $k n i$ mutant embryos. In order to visualize $k n i$ expression in kni mutant embryos, we made use of a transgene P4,4 kni-lac $Z$ (Pankratz et al., 1989) which drives $\beta$-galactosidase reporter gene expression under the control of the cis-acting sequences of the kni gene. $k n i^{\mathrm{FCl} 13}$ heterozygous males containing the reporter gene transcript were crossed to heterozygous $k n i{ }^{\mathrm{FCl} 3}$ females. In order to identify the homozygous mutant $k n i^{\mathrm{FCl} 13}$ embryos that were derived from this cross, embryos were stained with both anti- $\beta$-galactosidase antibodies and anti-even-skipped antibodies (Frasch et al., 1987). In the absence of kni activity based on the even-skipped expression pattern, heterozygous $k n i^{\mathrm{FCl} 3}$ or wildtype embryos (a) can be distinguished from homozygous $k n i^{\mathrm{FCl} 3}$ embryos (c). Note that the homozygous $k n i{ }^{\mathrm{FCl3}}$ embryo shown in (c) does not contain the $\beta$-galactosidase reporter gene construct and lacks the even-skipped stripe 5 and 6 of expression. (b) Heterozygous $k n i^{\mathrm{FCl} 13}$ or wildtype embryo expressing $\beta$-galactosidase in a region which overlaps even-skipped stripe 4 to 6 of expression. Note that the anterior cap domain (arrowhead) and the posterior expression domain of the reporter gene construct show similar signal intensities. (d) Homozygous $k n i^{\mathrm{FCl} 3}$ embryo containing the $\beta$-galactosidase reporter gene. Note that the posterior expression domain is strongly enhanced over the expression level in the anterior cap domain (arrowhead).

posterior domain of kni expression is essential for abdomen formation in the embryo. In contrast, $k n r l$ lacks this function completely. As shown recently, the knrl gene contains about $19 \mathrm{~kb}$ of intron sequences. Due to the size of the transcription unit (about $23 \mathrm{~kb}$ in length) and the rapid nuclear divisions in the early Drosophila embryo, the primary knrl transcript cannot be transcribed in full length and becomes aborted through the rapid mitotic cycles during the phenocritical period in the embryo (Rothe et al., 1992). The adjustment of $k n i$ to these rapid mitotic divisions is not only reflected in the small size of the $k n i$ transcription unit ( $3 \mathrm{~kb}$ ), but also by a different set of transacting factors as compared with $\mathrm{knrl}$, which is non-functional in the posterior region of the blastoderm embryo (Rothe et al., 1992). For both genes $\mathrm{Kr}$ activity is directly or indirectly required for activation of expression. As the $K r$ protein forms a concentration gradient in the centre of the embryo, a low level of $\mathrm{Kr}$ activity may be sufficient to activate $k n r l$ directly or indirectly. In the anterior, the low level of $h b$ activity overcomes $K r$-dependent activation by repression, thereby setting the anterior border of the $k n r l$ expression domain. Whether high levels of $K r$, which are known to cause repression (Licht et al., 1990; Sauer and Jäckle, 1993), contribute to the anterior restriction of the $k n r l$ expression domain remains to be shown. No repressor appears to be required for the establishment of the posterior border of this expression domain. Thus, a low level of $K r$ activity may provide a critical threshold concentration for $K r$-dependent activation which sets the posterior limit of the $k n r l$ posterior domain. kni activity, once accumulated, limits the level of knrl expression by suppression and eventually turns off the gene during early gastrulation. This basic transacting factor requirement is shared by kni. In addition, however, $k n i$ is activated by a global activator system and the spatial limit of $k n i$ gene expression requires a repressor system, provided by $t l l$ activity, which determines the posterior border of the kni expression domain. Finally, kni transcription is directed by a RNA-polymerase II which, in contrast to knrl, contains the $140 \mathrm{kDa}$ subunit characterized by the wimp mutation. 


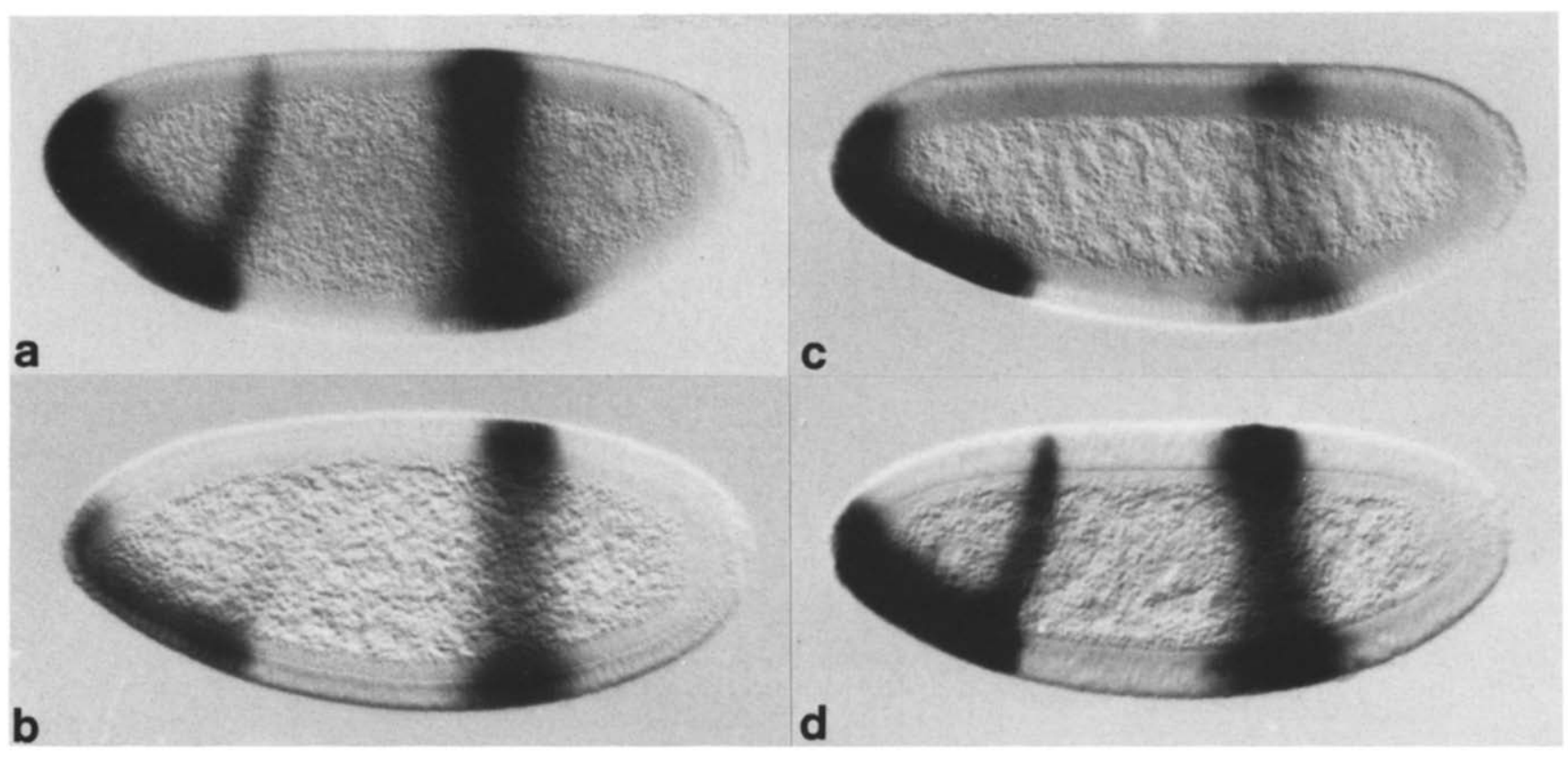

Fig. 6. Expression pattern of $k n i$ and $k n r l$ in embryos containing the wimp gene product. Wildtype embryos (a) and embryos laid by wimp/plus females (b-d) hybridized with kni cDNA (a and b) or the $k n r l$ cDNA (c and d). Note that kni expression is significantly reduced in embryos containing the wimp gene product while the expression of $k n r l$ is not affected by wimp. Thus, the mutant subunit of the RNA-polymerase II does not interfere with $k n r l$ expression but with the expression of $k n i$. As there is no proper internal control for kni expression in wildtype and wimp embryos, embryos were fixed and stained in parallel under identical conditions.

\subsection{Spatial control of the knrl and kni anterior cap domains}

In embryos derived from females mutant for the gene $b c d$, the cap expression domains of both $k n i$ and $k n r l$ are
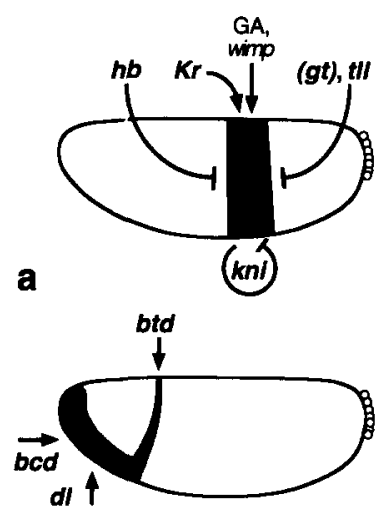

C

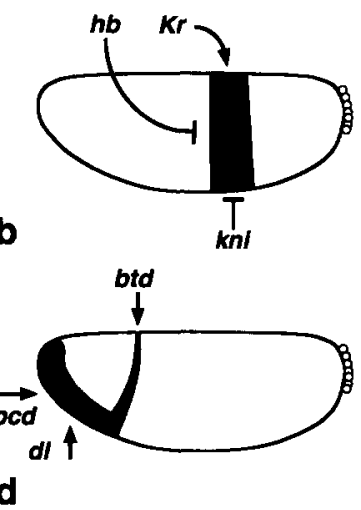

Fig. 7. Schematic representation of the transacting factor requirement

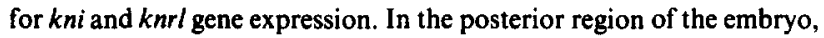
expression of $k n i$ (a) and $k n r l$ (b) is controlled by similar although not identical sets of regulators, whereas in the anterior region of the embryo the expression of $k n i$ (c) and $k n r l$ (d) requires identical sets of transactivators. Arrows represent activating activities, bars repressing activities of the indicated genes. GA stands for the yet unknown global activator system. $g t$ is put into brackets, as it is not clear if $g t$ actually represses $k n i$ in the wildtype situation. For details, see text. absent (Fig. 2c and h). In embryos derived from females carrying extra copies of the $b c d$ gene in their genome, which results in increased $b c d$ protein concentrations along the anterior-posterior axis (Driever and NüssleinVolhard, 1988), the ventral boundary of the cap expression domain of both genes is expanded in a $b c d$ protein concentration-dependent manner towards the posterior (Fig. 8). However, the dorsal boundary of the domain remains unchanged. No effect on the cap expression domains has been observed in embryos derived from females mutant for nanos (see Fig. 2a, b, $\mathrm{f}$ and g), the essential component of the posterior organizer system (Nüsslein-Volhard et al., 1987; Lehmann and NüssleinVolhard, 1991). In embryos derived from females mutant for the gene tor, which is required for development of the terminal regions (Klingler et al., 1988), no significant effect can be observed with respect to kni and knrl expression in the anterior cap domain when tor activity is absent (Fig. $2 \mathrm{~d}$ and i). However, in embryos which derived from females containing the dominant gain-offunction allele tor $^{\mathrm{D} 4021}$, the anterior cap domain is expanded posteriorly at the ventral side only (Fig. $2 \mathrm{e}$ and k).

As the cap domains display a dorsal-ventral asymmetry, we wanted to determine whether the maternal genes that establish dorsal-ventral polarity also affect $k n i$ and $k n r l$ expression. In embryos derived from females mutant for the gene $d l$, which fail to develop 


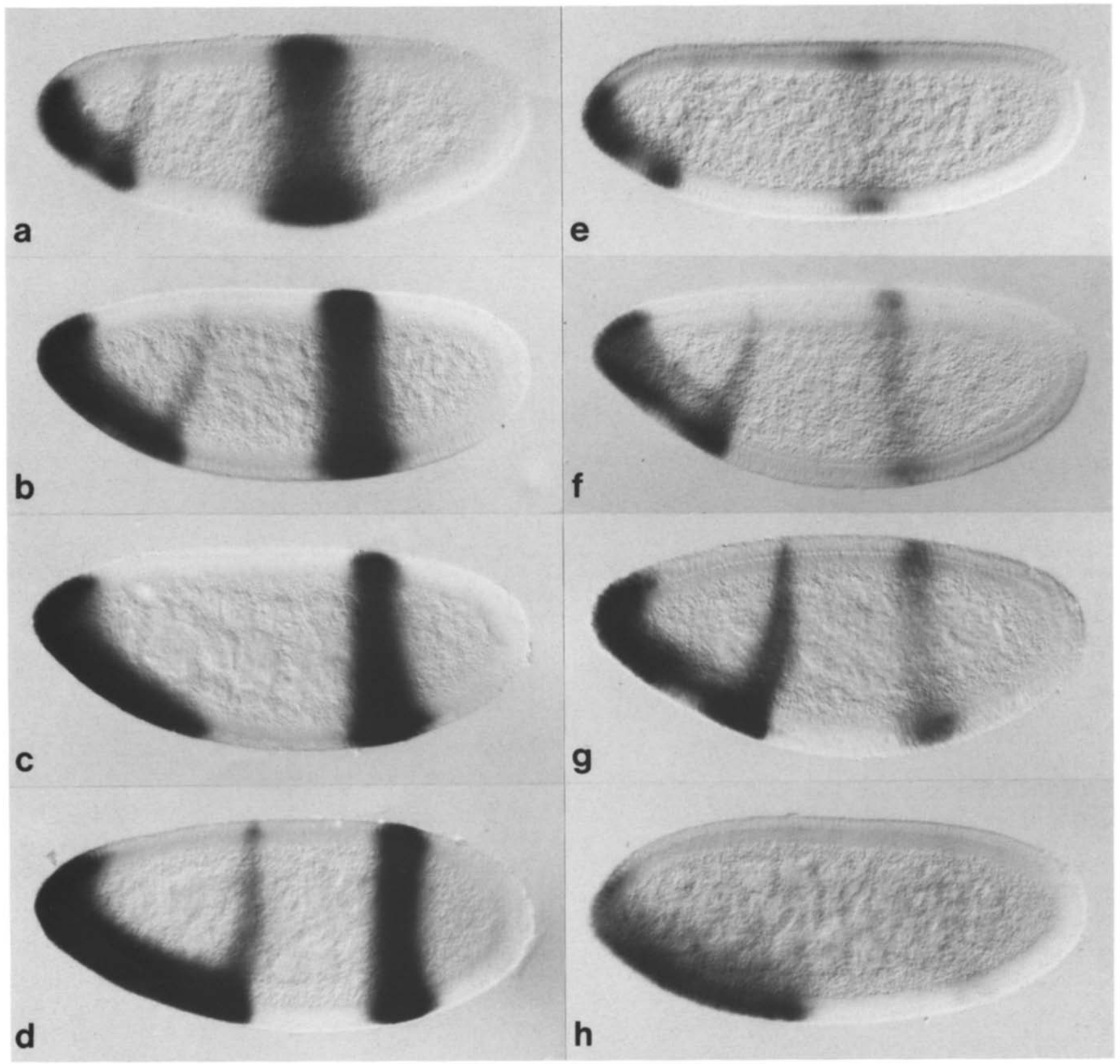

Fig. 8. Expression patterns of $k n i$ and $k n r l$ in $b c d$ variants. Embryos derived from females carrying 1 (a and e), 2 (wildtype) (b and $f$ ), 4 (c and $\mathrm{g})$, or $6(\mathrm{~d}$ and $\mathrm{h}$ ) functional copies of the $b c d$ gene have been hybridized with a $k n i \mathrm{cDNA}$ probe (a-d), or with a $k n r l \mathrm{cDNA}$ probe (f and $\mathrm{g}$ ). Note that the ventral boundary of both the $k n i$ and $k n r l$ anterior cap domains expands posteriorly in response to the increased bcd dosage, whereas the dorsal boundary is hardly affected. Since $b c d$ protein forms a concentration gradient in the embryo it is likely that at a certain threshold of $b c d$ concentration $b c d$ activity can directly or indirectly activate $k n i$ and $k n r l$ expression on the ventral side of the embryo (acting in concert with $d l$ activity; see text). Note that the anterior stripe domains also shift towards posterior in $b c d$ protein concentration-dependent manner.

ventral structures due to the lack of $d l$ activity (NüssleinVolhard et al., 1987), the anterior cap domain of the two genes is absent (Fig. 9a and c). Conversely, in embryos derived from females with a dominant mutation in the Toll (Tl) gene (Anderson et al., 1985) (where the $d l$ gene product is equally distributed in the nuclei along the dorsal-ventral axis of the embryo and embryos develop a completely ventralized mutant phenotype) the anterior cap domains of $k n i$ and $k n r l$ lose their asymmetry; they are expressed both dorsally and ventrally in those embryos (Fig. $9 \mathrm{~b}$ and d). These results indicate that $k n i$ and $k n r l$ are activated in response to $d l$ and $b c d$ activity, i.e. if either one of the two morphogens is missing, both genes fail to be expressed in the anterior cap domain. 


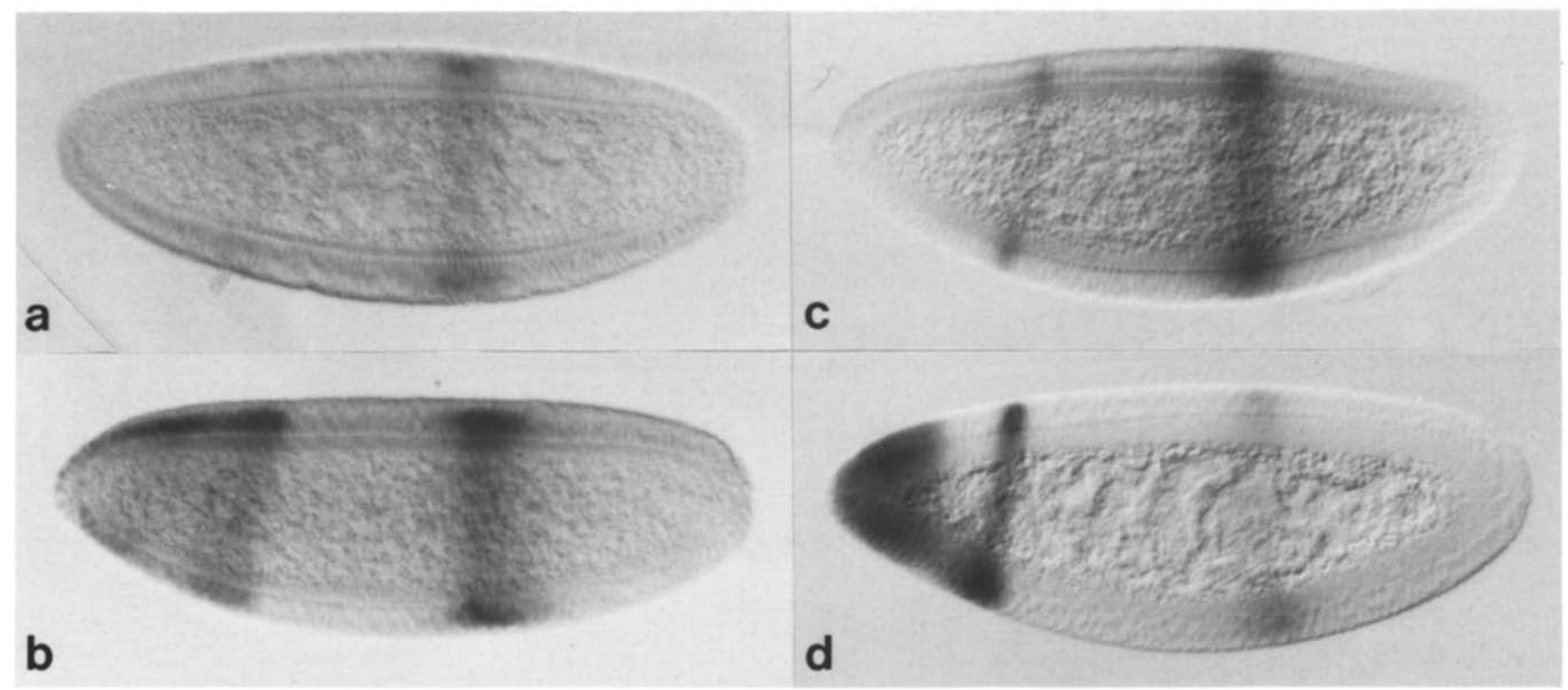

Fig. 9. Anterior cap domain of kni and knrl expression is controlled by maternal genes of the dorsal-ventral system. kni expression (a and b) is detected by antibody staining and $k n r l$ expression ( $\mathrm{c}$ and $\mathrm{d}$ ) by whole mount in situ hybridization. In embryos lacking $d l$ activity (a and c) $k n i$ and $k n r l$ fail to be expressed in their anterior cap domains. Embryos derived from females carrying a dominant mutation of $T l$ (b and d) express $k n i$ and $k n r l$ all around the anterior pole, therefore the anterior cap domains have lost their asymmetry. This shows that the formation of the anterior cap domains of $k n i$ and $k n r l$ is dependent on $d l$ activity.

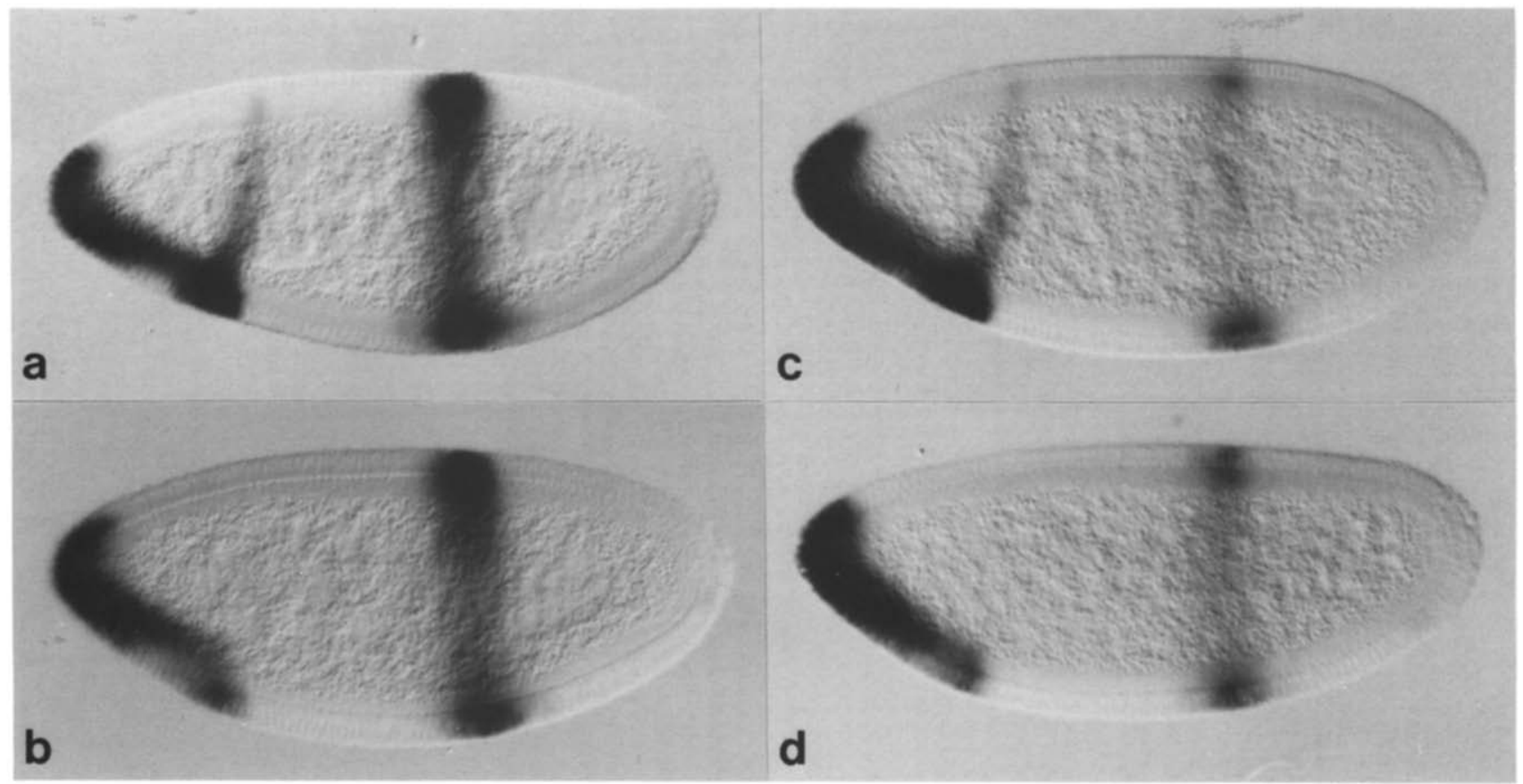

Fig. 10. $k n i$ and $k n r l$ expression in wildtype and btd ${ }^{\mathrm{XG}}$ mutant embryos. (a and b) $k n i$ expression and (c and d) $k n r l$ expression visualized by whole mount in situ hybridization as described in the legend of Fig. 1 . The anterior stripe expression domains of $k n i$ and $k n r l$ form during the late stage of blastoderm cellularization ( $\mathrm{a}$ and $\mathrm{c}$ ), whereas these domains are not detected in embryos mutant for the head specific segmentation gene bid (b and d). Note that only the anterior stripe domain, not the anterior cap domain, is affected in both cases. 
Since both $b c d$ and $d l$ encode transcription factors (St. Johnston and Nüsslein-Volhard, 1992; for review), they could activate $k n i$ and $k n r l$ expression directly, or their activity could be mediated by zygotic target genes which act in the corresponding region of the embryo. We therefore analysed the expression patterns of the two genes in embryos mutant for the $b c d$-dependent gap-like segmentation genes which act in the head region of the embryo. Embryos homozygous for mutant alleles of the genes orthodentical (otd), empty spiracles (ems), or buttonhead (btd) (Cohen and Jürgens, 1990) show a normal anterior cap domain of expression (Fig. 10; data for otd and $e m s$ are not shown). Furthermore, the known zygotic target genes of $d l$ such as snail and twist (St. Johnston and Nüsslein-Volhard, 1992; for review) had no effect on the anterior cap expression domains of $k n i$ and $k n r l$ (data not shown). Thus, it appears likely that the proper establishment of the identical $k n i$ and $k n r l$ anterior cap domains of expression is controlled by the combined $b c d$ and $d l$ activities directly. The $b c d$ and $d l$ gene products form an anterior to posterior concentration gradient and a dorsal to ventral nuclear localization gradient, respectively. We speculate that both $k n i$ and $k n r l$ are activated in response to the combined action of the two maternal morphogens in the anterior-ventral position of the embryo and that each morphogen determines the spatial limits of gene expression. This suggests a model that both $d l$ and $b c d$ protein provide concentration thresholds above which, in the presence of appropriate concentrations of the other gene product, the kni and $k n r l$ genes can be activated. Such a situation, where the combined action of the anterior and the ventral morphogens is required for activation of a zygotic gene, is unprecedented and it will be interesting to learn how this interaction is achieved molecularly.

\subsection{Spatial control of kni and knrl anterior stripe domains}

The anterior stripes of $k n i$ and $k n r l$ can first be detected in the late phase of blastoderm cellularization shortly after the anterior caps have been formed (Fig. 1). Like the anterior caps, the anterior stripes are dependent on the anterior maternal organizer system. In embryos from homozgous $b c d$ mutant females the anterior stripe domains are missing (Fig. 2c and h). However, in contrast to the anterior caps, the anterior stripe domains are not activated by the $b c d$ gene product directly. $b c d$ function is mediated in the embryonic head through the gaplike segmentation genes ems, otd, and btd (Cohen and Jürgens, 1990). The anterior stripes of kni and knrl at $70 \% \mathrm{EL}$ lie in the expression domain of $b t d(65-77 \% \mathrm{EL}$, Wimmer et al., 1993) and in btd mutant embryos the anterior stripe domains cannot be observed (Fig. 10) (Cohen and Jürgens, 1990). This suggests that btd activates directly or indirectly the expression of $k n i$ and $k n r l$. Since the btd stripe is wider than the anterior stripes of $k n i$ and $k n r l$, other genes must also be involved in the regulation of these expression domains. However, neither otd, ems, nor any of the other known gap genes that are expressed in the head region $(t l l, g t, h b)$ affect the anterior stripe domains (Fig. 3, data for otd, ems and $g t$ not shown). Therefore it remains unclear how the expression of $k n i$ and $k n r l$ in this anterior stripe domain is delimited to such a thin band. Either repression of the anterior stripe expression at the edges of the $b t d$ domain is redundant, or some as yet unidentified genes are needed to repress, or the btd gene needs one or more spatially restricted coactivators in order to be able to activate $k n i$ and $k n r l$.

\section{Conclusions}

$k n i$ and $k n r l$ are expressed within the same spatial limits in the blastoderm embryo. Only one of the three expression domains can be functionally assigned by the $k n i$ mutant phenotype. $k n i$ activity in the posterior domain is required for abdominal segmentation during a short phenocritical period around blastoderm stage, while $k n r l$ expression in the same domain is nonfunctional, although $k n r l$ can provide partial $k n i$ activity when expressed from an intron-lacking minigene (Rothe et al., 1992). This finding suggested that both genes derive from a common ancestral gene; $k n i$ had adjusted to the very rapid mitotic divisions during the early stages of Drosophila development while the $k n r l$ primary transcript is too long to be fully transcribed during the phenocritical period for abdominal segmentation (Rothe et al., 1992). In addition, kni has gained additional control for efficient transcription in the posterior domain which might be reflected in the as yet unknown global activator (Pankratz et al., 1992), in the sensitivity to the wimp mutation and the posterior repression system which are not functional in the control of $k n r l$ (Fig. 6). Alternatively, the mode of regulation described for kni might be the evolutionarily conserved control mechanism and what we envisage, with the reduced set of factors involved in the regulation of $k n r l$, might represent a secondary reduction due to the lack of function of the gene in the posterior domain. In this view, the identical transacting requirement for $k n i$ and $k n r l$ anterior expression suggests evolutionary constraints for their control because both genes are functionally conserved in the anterior expression domains. However, there is no obvious phenotype associated with the lack of $k n i$ function in the anterior region of the embryo. In addition, several mutagenesis screens have failed so far to isolate a $k n r l$-specific mutation (unpublished data). This leaves the possibility that $k n i$ and $k n r l$ carry redundant or very similar functions in the two anterior domains of expression. We note, however, that the lack of both gene activities due to chromosomal deficiencies causes, in addition to the kni segmentation 
phenotype, a strong head defect that remains to be analysed in detail.

An interesting result is that the anterior cap domains fail to be expressed in the absence of either $b c d$ or $d l$ activity. This suggests that it is the combined action of the two morphogens which activates $k n i$ and $k n r l$ gene expression in the anterior cap domain. This mode of control, by genetic means, is reminiscent of the finding that $d l$-dependent activation of gene expression in the lateral region of the blastoderm embryo depends on cooperative DNA binding interactions between $d l$ protein and a helix-loop-helix-type transcription factor (Jiang and Levine, 1993). The possibility of a similar mechanism required for the activation of both $k n i$ and $k n r l$ in the anterior domain would imply that both genes contain a cis-acting element that serves as a template to bring the two morphogens into close proximity, so they can help each other bind and function in combination to activate gene expression at peak levels.

\section{Materials and methods}

\subsection{Genetics and expression pattern analysis}

Mutant lines such as bicoid ${ }^{\mathrm{EI}}$, hunchback ${ }^{9 \mathrm{Q}}$, nanos ${ }^{\mathrm{L} 7}$, torso $^{\mathrm{PM}}$, torso ${ }^{\mathrm{D} 4021}$, Krüppel ${ }^{\mathrm{I}}$, knirps ${ }^{\mathrm{FCl3}}$, Df(3L)ri ${ }^{\mathrm{XTI}}$, tailless $^{\mathrm{g}}$, giant and $b t d^{\mathrm{XG}}$ are described in Tearle and Nüsslein-Volhard (1987); females containing additional copies of the bicoid gene were obtained from the $b c d^{+5} b c d^{+8} /$ FM7; +/+ strain (Driever and NüssleinVolhard, 1988). Embryos were either identified by the genotype of the mother, by doublestaining with the corresponding molecular probes or by alterations of expression patterns in a quarter of embryos in the case of zygotic mutants. In situ hybridization to whole mount preparations of embryos was done according to Tautz and Pfeifle (1989), antibody staining with anti-knirps and anti-eve protein antibodies (gifts of N. Gerwin and M. Levine) of whole mount embryos was carried out as described (Macdonald and Struhl, 1986) using the Vectastain ABC Elite horseradish peroxidase system. Expression of the transgene $\mathrm{P} 4,4 \mathrm{kni}$-lac $Z$ was monitored by $\beta$-galactosidase antibody staining as described in Pankratz et al. (1992).

\section{Acknowledgements}

We thank D. Ish-Horowicz for the wimp mutant, $M$. Levine for the anti-eve antibodies, and $\mathrm{C}$. NüssleinVolhard for various mutant strains. We thank our colleagues in the lab for critical discussions and Mrs E. Vet- ter for preparing the manuscript. The work was supported by the Max-Planck Society, the Deutsche Forschungsgemeinschaft and the Fonds der Chemischen Industrie. M. Rothe, E.A. Wimmer and M. GonzálezGaitán are fellows of the Fonds der Chemischen Industrie, the Boehringer Ingelheim Fonds, and the EC program 'Human Capital and Mobility', respectively.

\section{References}

Anderson, K.V., Bokla, L. and Nüsslein-Volhard, C. (1985) Cell 42, 791-798.

Capovilla, M., Eldon, E.D. and Pirrotta, V. (1992) Development 114, 99-112.

Cohen, S.M. and Jürgens, G. (1990) Nature 346, 482-485.

Driever, W. and Nüsslein-Volhard, C. (1988) Cell 54, 95-104.

Eldon, E. and Pirrotta, V. (1991) Development 111, 367-378.

Frasch, M., Hoey, T., Rushlow, C., Doyle, H. and Levine, M. (1987) EMBO J. 6, 749-759.

Howard, K. (1990) Semin. Cell Biol. 1, 161-172.

Ingham, P. (1988) Nature 335, 25-34.

Jiang, J. and Levine, M. (1993) Cell, 72 741-752.

Klingler, M., Erdelyi, M., Szabad, J. and Nüsslein-Volhard, C. (1989) Nature 335, 275-277.

Kraut, R. and Levine, M. (1991) Development 111, 601-609.

Lehmann, R. and Nüsslein-Volhard, C. (1991) Development 112, 679-691.

Licht, J.D., Gossel, M.J., Figge, I. and Hansen, U. (1990) Nature 346, 76-79.

Macdonald, P. and Struhl, G. (1989) Nature 324, 537-545.

Nauber, U., Pankratz, M.J., Kienlin, A., Seifert, E., Klemm, U. and Jäckle, H. (1988) Nature 336, 489-492.

Nüsslein-Volhard, C. and Wieschaus, E. (1980) Nature 287, 795-801.

Nüsslein-Volhard, C., Frohnhöfer, H.G. and Lehmann, R. (1987) Science 238, 1675-1681.

Oro, A.E., Ong, E.S., Margolis, J.S., Posakony, J.W., McKeown, M. and Evans, R.M. (1988) Nature 336, 493-496.

Pankratz, M.J., Hoch, M., Seifert, E. and Jäckle, H. (1989), Nature 341, 337-340.

Pankratz, M.J., Busch, M., Hoch, M., Seifert, E. and Jäckle, H. (1992) Science 255, 986-989.

Pankratz, M.J. and Jäckle, H. (1993) In: Development of Drosophila. Cold Spring Harbor Laboratory Press, 467-516.

Parkhurst, S.M. and Ish-Horowicz, D. (1991) Genes Dev. 5, 341-357.

Rothe, M., Nauber, U. and Jäckle, H. (1989) EMBO J. 8, 3087-3094.

Rothe, M., Pehl, M., Taubert, H. and Jäckle, H. (1992) Nature 359, $156-159$.

Sauer, F. and Jäckle, H. (1993) Nature 364, 454-457.

Steingrimsson, E., Pignoni, F., Liaw, G.J. and Lengyel, J.A. (1991) Science 254, 418-421.

St. Johnston, D. and Nüsslein-Volhard, C. (1992) Cell 68, 201-209.

Tautz, D. and Pfeifle, C. (1989) Chromosoma 98, 81-85

Tearle, R. and Nüsslein-Volhard, C. (1987) Dros. Info. Ser. 66, 209-269.

Weigel, D., Jürgens, G., Küttner, F., Seifert, E. and Jäckle, H. (1989) Cell 57, 645-658.

Wimmer, E.A., Jäckle, H, Pfeifle, C. and Cohen, S.M. (1993) Nature $366,690-694$. 